\title{
Control of Deuteron Ions to Escape from the Magnetic Mirror Bottle by a Perpendicular Supplemental Mirror
}

\author{
Mitsuaki Nagata \\ Soft Creator Company, Shinmachi, Nakagyo-ku, Kyoto, Japan \\ Email: nagata@heian-kogyo.jp
}

How to cite this paper: Nagata, M. (2019) Control of Deuteron Ions to Escape from the Magnetic Mirror Bottle by a Perpendicular Supplemental Mirror. Journal of Modern Physics, 10, 1394-1400. https://doi.org/10.4236/jmp.2019.1011092

Received: June 28, 2019

Accepted: October 26, 2019

Published: October 29, 2019

Copyright $\odot 2019$ by author(s) and Scientific Research Publishing Inc. This work is licensed under the Creative Commons Attribution International License (CC BY 4.0).

http://creativecommons.org/licenses/by/4.0/

\section{c) (i) Open Access}

\begin{abstract}
We have inquired into a means to decrease the number of charged particles escaping from a loss cone of a magnetic mirror reactor as many as possible. We previously reported the way of installing a supplemental magnetic mirror (which has a cyclotron heating space within) at the exit of a main magnetic bottle. The cyclotron heating space was set to increase a reflection-efficiency of the supplemental mirror. We could not suppress the loss of the escaping deuteron ions sufficiently even with a very long mirror and a very powerful electric field. Then, in this work we propose a new plan of installing another supplemental mirror besides the previous supplemental mirror. A new mirror is set perpendicularly to the center axis of the main bottle. By the addition of the perpendicular mirror, an efficiency of sending back of escaping deuteron ions is considerably theoretically improved. Also in the previous work, since we did not touch how to supply a high-frequency electric field to the cyclotron heating space, here we consider supplying it by an extraordinary-wave with a cyclotron frequency. It is mentioned that propagation of an extraordinary-wave with an electron cyclotron frequency depends on a magnetic field strength and density of escaping electrons.
\end{abstract}

\section{Keywords}

Magnetic Mirror Reactor, Control of Escaping Deuteron Ions, Electron and Deuteron Ion Heating by Extraordinary Waves

\section{Introduction}

It is considered that the most sincere problem in research with respect to a fusion reactor of magnetic mirror-type is that escaping loss of charged particles from the loss cone is still too much [1]. We first examined a means [2] of setting 
a supplemental magnetic mirror (with a cyclotron resonance space within) at the exit of a main magnetic bottle. The principal aim in Ref. [2] was to reduce the half-vertical angle (called the loss angle) of the loss cone by the cyclotron resonance heating [3]. However, we could not sufficiently suppress the loss of escaping deuteron ions even with a very powerful electric field and a very long magnetic mirror. So, we proposed introduction of a means for replenishing a large quantity of deuteron ions from the outside. But in this work, we mention an idea that, by setting another supplemental magnetic mirror (called Perp-mirror) perpendicularly to the center line of the supplemental mirror (called $\mathrm{Pa}$ ra-mirror) which is set in parallel with the center line of the main bottle, we may be able to induce "most of charged particles escaping from the exit (plane $d_{1} d_{0} d_{4}$ in Figure 1) of Para-mirror" outside the loss cone of Perp-mirror. Based on this idea, we mention in $\$ 3$ a new plan to reclaim escaping charged particles themselves.

We did not touch in Ref. [2] how to supply a high frequency electric field to the cyclotron resonance heating space. Then, in this work we consider transmitting an extraordinary wave with an electron cyclotron frequency or with a cyclotron frequency for a deuteron ion $\mathrm{D}^{+}$to a heating space. It is mentioned in $\$ 2$ that propagation of an extraordinary wave with an electron cyclotron frequency depends on plasma density and magnetic field strength.

\section{Heating by an Extraordinary Wave}

The refractive index $n_{x}$ [4] [5] [6] [7] for an extraordinary wave (called $\mathrm{X}$-wave) with a frequency $\omega$ is given by

$$
n_{x}^{2}=\frac{\left(1-\beta_{i}^{2}\right)\left(1-\beta_{e}^{2}\right)-2 \alpha^{2}\left(1-\beta_{i} \beta_{e}\right)+\alpha^{4}}{\left(1-\beta_{i}^{2}\right)\left(1-\beta_{e}^{2}\right)-\alpha^{2}\left(1-\beta_{i} \beta_{e}\right)}
$$

Here,

$$
\beta_{e}=\omega_{c} / \omega, \quad \beta_{i}=\omega_{i} / \omega,
$$

$\omega_{c} \simeq q B / m_{e}$, an electron cyclotron frequency

( $B$ : a magnetic field strength, $-q$ : the electron charge, $m_{\mathrm{e}}$ : the rest mass of an electron),

$\omega_{i} \simeq q B / m_{i}$, a cyclotron frequency for a deuteron ion $\mathrm{D}^{+}$(a deuteron mass $\left.m_{i} \simeq 3680 m_{e}\right)$,

$$
\alpha^{2}=\left(\frac{\omega_{p e}}{\omega}\right)^{2}+\left(\frac{\omega_{p i}}{\omega}\right)^{2}
$$

$\omega_{p e}=\left(\frac{n_{e} q^{2}}{m_{e} \varepsilon_{0}}\right)^{1 / 2}$ a plasma frequency for electrons ( $n_{e}$ : electron density, $\varepsilon_{0}$ : the dielectric constant of vacuum),

$$
\omega_{p i}=\left(\frac{n_{i} q^{2}}{m_{i} \varepsilon_{0}}\right)^{1 / 2} \text { a plasma frequency for ions }\left(n_{i} \text { : deuteron ion density }=n_{e}\right)
$$




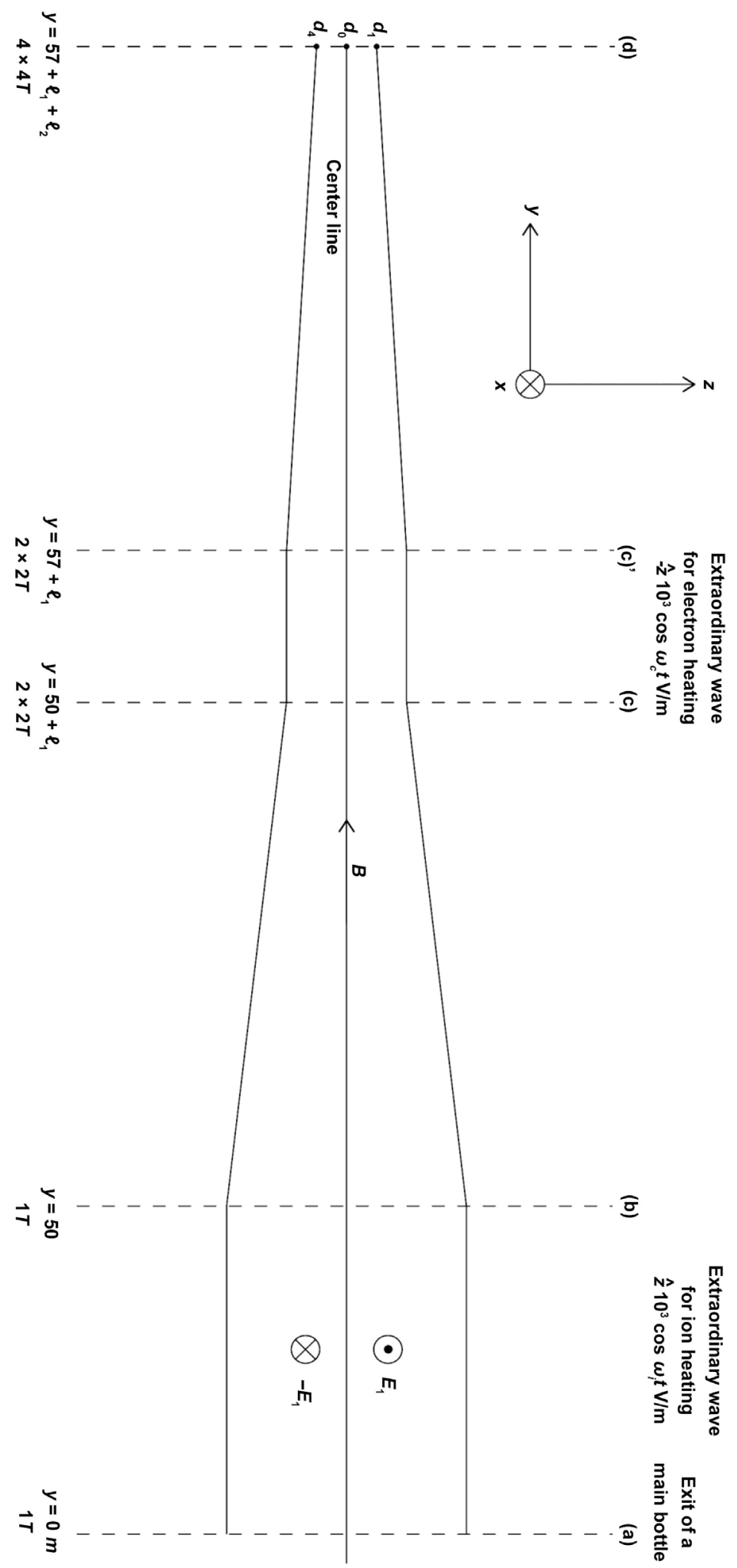

Figure 1. A supplemental magnetic mirror (called Para-mirror) for reclaiming charged particles to escape from the exit (plane (a)) of the main bottle. Constant electric fields $\pm E_{1} \mathrm{~V} / \mathrm{m}$ are for making charged particles go near the central plane perpendicular to the $\mathrm{z}$-axis, $\boldsymbol{B}$ is a magnetic field which is a function of position. 
We examine whether two kinds of X-wave with frequencies $\omega_{c}$ and $\omega_{i}$ can pass through a plasma.

\subsection{About X-Wave with $\omega=\omega_{c}$}

Since $\beta_{e}=1$ and $\beta_{i}=\omega_{i} / \omega_{c}=m_{e} / m_{i} \ll 1$ we have

$$
n_{x}^{2} \simeq \frac{-2 \alpha^{2}+\alpha^{4}}{-\alpha^{2}}=2-\alpha^{2}>0 \text { or } \alpha^{2}<2
$$

When a value of $B$ is set, an upper limit of $n_{e}$ is determined from (2). We consider a supplemental mirror (Para-mirror) as shown in Figure 1. X-wave with $\omega=\omega_{c}$ is supplied between planes (c) and (c)'. Then, we have

$$
\begin{gathered}
B=4 \mathrm{~T}, \\
\omega_{c}=0.704 \times 10^{12} \mathrm{sec}^{-1}, \\
\alpha^{2} \simeq\left(\omega_{p e} / \omega_{c}\right)^{2}<2 \text { or } \omega_{p e} \lesssim 10^{12}, \therefore n_{e} \lesssim 3 \times 10^{20} \mathrm{~m}^{-3} .
\end{gathered}
$$

Since it is presumed that a target density for electron density in a main bottle is $10^{21} \mathrm{~m}^{-3}$, we have set an electron heating space behind mirror (b)-(c).

\subsection{About X-Wave with $\omega=\omega_{i}$}

We set a heating space for deuteron ions $\mathrm{D}^{+}$between planes (a)-(b). Since $\alpha^{2}>2, \beta_{i}=1$ and $\beta_{e}=\omega_{c} / \omega_{i}=m_{i} / m_{e} \gg 1$, we have from (1)

$$
n_{x}^{2} \simeq \frac{2 \alpha^{2} \beta_{e}+\alpha^{4}}{\alpha^{2} \beta_{e}}>0
$$

There is no essential restriction for values of $\alpha^{2}$ and $\beta_{e}$.

\section{Sending Back of Escaping Deuteron Ions by a Perpendicular Mirror}

Even if Para-mirror of such a large scale as shown in Figure 1 is used, it is clear, based on the examination in Ref. [2], that the escaping loss of charged particles is still too large. Then, we propose a means of installing another magnetic mirror (Perp-mirror) perpendicularly to the center line of the Para-mirror, as shown in Figure 2. Using Perp-mirror, we plan to reclaim charged particles themselves escaping from Para-mirror. In Figure 2, plane $d_{1} d_{0} d_{4}$ is the exit of $\mathrm{Pa}$ ra-mirror. It is assumed that most of the magnetic force lines coming out of plane $d_{1} d_{0} d_{4}$ enter into surfaces $a_{1} a_{2}$ and $a_{3} a_{4}$ of the ferromagnetic substance. Our plan to reclaim deuteron ions (called the test ions) escaping from plane $d_{1} d_{0}$ and going to surface $a_{1} a_{2}$ is through the following procedure:

1) Under the force in the direction of $\boldsymbol{E}_{1} \times \boldsymbol{B}_{1} \quad\left(\boldsymbol{E}_{1}\right.$ : a constant electric field, $\boldsymbol{B}_{1}$ : a magnetic field which is a function of position), the test ions get out of region $d_{1} d_{0} a_{2} a_{1}$, pass through plane $a_{2} a_{3}$ and go to plane $b_{2} b_{3}$. Here, it is expected that each incident angle of the test ions when passing plane $b_{2} b_{3}$ (each inclination from the direction parallel to the centerline) is considerably smaller than $90^{\circ}$. 


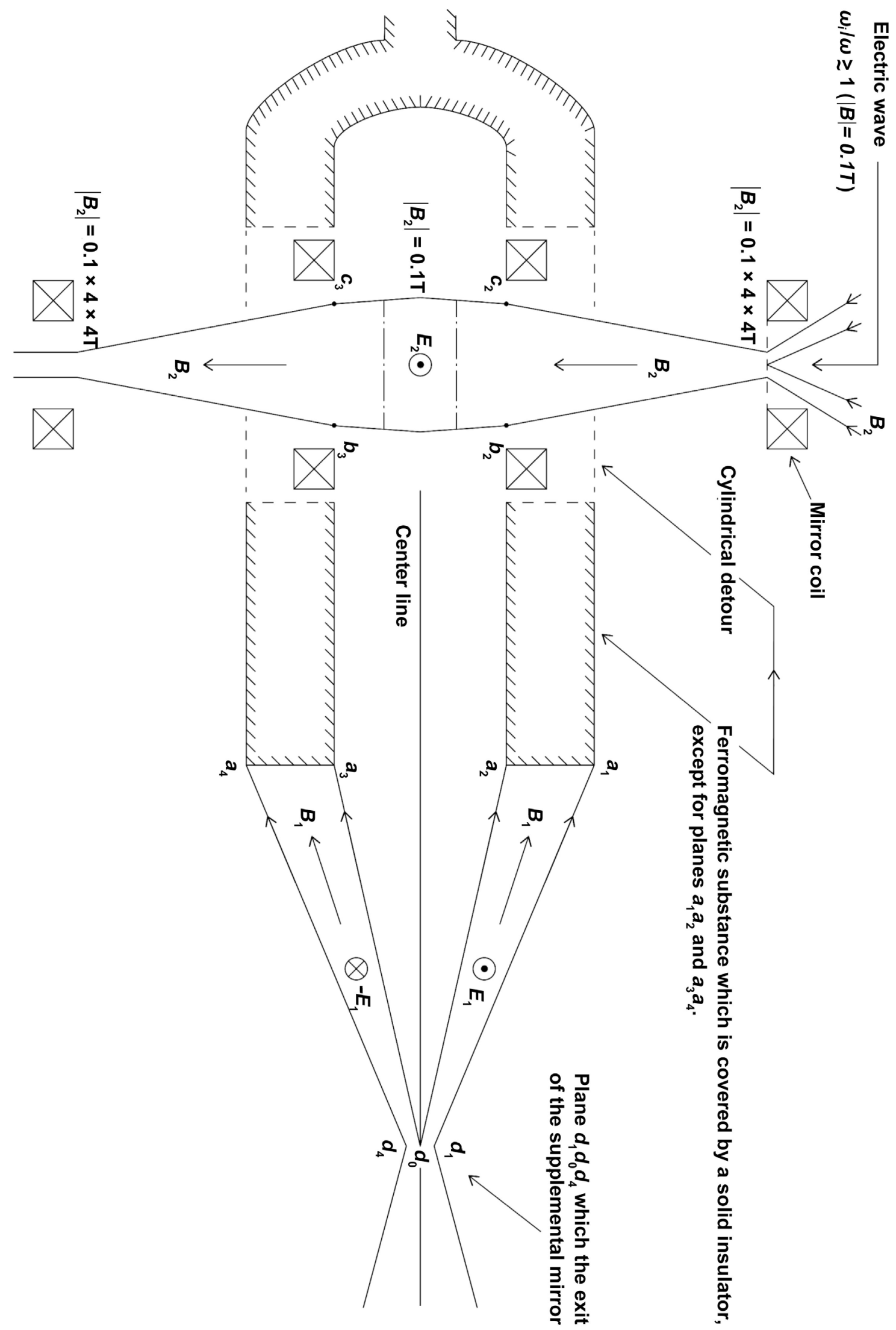

Figure 2. A schematic diagram of an apparatus (called Perp-mirror) to reclaim the escaping deuteron ions. 
2) Magnetic fields in the central part and the exit of Perp-mirror are $0.1 \mathrm{~T}, 0.1$ $\times 4 \times 4 \mathrm{~T}$, respectively. The loss angle of this mirror is $14.5^{\circ}$. Then, the test ions having entered within region $b_{2} b_{3} c_{3} c_{2}$ will begin roundtrip motions between the upper and lower reflection-parts of Perp-mirror.

3) In the above situation, when a constant electric field $\boldsymbol{E}_{2}$ is supplied within the central part $b_{2} b_{3} c_{3} c_{2}$, as shown in Figure 2, the test ions drift in the direction of $\boldsymbol{E}_{2} \times \boldsymbol{B}_{2} \quad\left(\boldsymbol{B}_{2}\right.$ : a magnetic field which is a function of position) and finally jump out in the right-hand side of plane $b_{2} b_{3}$. These test ions are expected to return to plane $d_{1} d_{0} d_{4}$. It should be noted that a part of particles may collide with planes $a_{1} a_{2}, a_{3} a_{4}, a_{2} b_{2}, a_{3} b_{3}$ and may become loss particles.

Since it can be expected that deuteron ions will not rush to the exits of Perp-mirror, an electric wave can be transmitted in parallel with the center line of Perp-mirror, by diverting aside the directions of the strong magnetic force lines from the mirror axis, as shown in Figure 2. The wave with the frequency $\omega$ which satisfies $\omega_{i} / \omega \gtrsim 1 \quad\left(\omega_{i}=q\left|\boldsymbol{B}_{2}\right| / m_{i},\left|\boldsymbol{B}_{2}\right|=0.1 \mathrm{~T}\right)$ can pass through Perp-mirror. The left-circularly polarized wave contributes to heating of deuteron ions. From the examination in Ref. [2], in order to shorten the length between planes (a) and (b) in Figure 1 but to heat heavy deuteron ions sufficiently, another heating region is necessary.

In this plan, the troublesome problem is that a necessary magnitude for $\boldsymbol{E}_{1}$ and a necessary length for $d_{0} a_{2}$ are extremely large. A ratio of $\left|\boldsymbol{E}_{1} \times \boldsymbol{B}_{1}\right| / \boldsymbol{B}_{1}^{2}\left(\boldsymbol{E}_{1} \perp \boldsymbol{B}_{1}\right)$ to the mean thermal velocity $\bar{v}_{i}\left(\simeq 2 \times 10^{6} \mathrm{~m} / \mathrm{s}\right)$ of deuteron ions at temperature $4 \times 10^{8} \mathrm{~K}$ is given by, for instance, when $\left|\boldsymbol{B}_{1}\right|=16 \mathrm{~T}$ and $\left|\boldsymbol{E}_{1}\right|=20 \times 10^{4} \mathrm{~V} / \mathrm{m}$,

$$
\frac{\left|E_{1}\right|}{\left|B_{1}\right|}: \bar{v}_{i}=\frac{20 \times 10^{4}}{16}: 2 \times 10^{6}=1: 160
$$

Even if such a powerful electric field is used, a necessary length for $d_{0} a_{2}$ is about $160\left(d_{1} d_{0}\right)$. However, this problem can be solved by setting constant electric fields $\pm \hat{x}\left|\boldsymbol{E}_{1}\right|$ also in the space with the smaller magnetic field $1 \mathrm{~T}$ between planes (a)-(b) in Figure 1.

\section{Conclusions}

We have mentioned the means for sending back most of escaping charged particles to the main bottle by the help of Para-mirror (Figure 1) and Perp-mirror (Figure 2). An efficiency of sending back depends on whether charged particles escaping from plane $d_{1} d_{0} d_{4}$ can pass through plane $b_{2} b_{3}$ or not. As a simple countermeasure, it is considered to make $\left|\boldsymbol{E}_{1}\right|$ between planes $d_{1} d_{4}$ and $a_{1} a_{4}$ smaller and instead to make $\left|E_{1}\right|$ between planes (a) and (b) of Figure 1 bigger. Also, it is necessary to decrease the number of charged particles escaping from plane $d_{1} d_{0} d_{4}$ as many as possible, because scatterings (collisions) by the Coulomb forces ought to obstruct an orderly drift-movement in the direction of $\boldsymbol{E}_{1} \times \boldsymbol{B}_{1}$.

Further, we consider that charged particles heating should be done outside the 
main bottle in order not to disturb the stability of a plasma within the main bottle. Both Para-mirror and Perp-mirror are heaters to warm charged particles very slowly.

\section{Conflicts of Interest}

The author declares no conflicts of interest regarding the publication of this paper.

\section{References}

[1] Ågren, Å.O. and Moiseenko, V.E. (2014) Plasma Physics and Controlled Fusion, 56, Article ID: 095026.

[2] Nagata, M. and Sawada, K. (2019) Journal of Modern Physics, 10, 145-156.

[3] Ivanov, A.A., Burdakov, A.V. and Bagryansky, P.A. (2015) Fusion Science and Technology, 68, 56.

[4] Sitenko, A.G. and Stepanov, K.N. (1957) Journal of Experimental and Theoretical Physics, 4, 512.

[5] Linhart, J.G. (1960) Plasma Physics. North Holland, Amsterdam.

[6] Stix, T.H. (1962) The Theory of Plasma Waves. McGraw-Hill, New York.

[7] Allis, W.P., Buchsbaum, S.J. and Bers, A. (1963) Waves in Anisotropic Plasmas. MIT Press, Cambridge, Mass. 